\title{
Comparative Review
}

\section{e Modified K-Hole Percutaneous Endoscopic Surgery for Cervical Foraminal Stenosis: Partial Pediculectomy Approach}

\author{
Chang-Ming Xiao, MD ${ }^{1,4}$, Ke-Xiao Yu, MD²,4, Rui Deng, MD ${ }^{3,4}$, Qing-Yan Long, MB ${ }^{3,4}$, \\ Lei Chu, MD ${ }^{3,4}$, Yang Xiong, $\mathrm{MD}^{3,4}$, Bei Sun, MD, ${ }^{3,4}$ Liang Chen, MD, PhD ${ }^{3,4}$, \\ Zheng-Jian Yan, $\mathrm{MD}^{3,4}$, and Zhong-Liang Deng, MD, $\mathrm{PhD}^{3,4}$
}

From: ${ }^{1}$ The Department of Spine Surgery and Bone Oncology, Hospital (T.C.M) Affliated To Southwest Medical University, No.182, Chunhui Road, Luzhou, Sichuan, China; ${ }^{2}$ Chongqing Hospital of Traditional Chinese Medicine, Chongqing, China; ${ }^{3}$ Department of Orthopedics,

The Second Affiliated Hospital of

Chongqing Medical University, Chongqing, China; ${ }^{4}$ Geriatric Clinical Research Center of

Chongqing, Chongqing, China.

Address Correspondence: Zhong-Liang Deng, MD, PhD Department of Orthopedics The Second Affiliated Hospital of Chongqing Medical University Linjiang Road 76\# Yuzhong District City Chongqing, Chin E-mail: zhongliang.deng@qq.com

Disclaimer: Chang-Ming Xiao and Ke-Xiao Yu contributed equally to this work and should be considered co-first authors. The study was supported by the National Nature Science Foundation of China (no. 81672230), the Health and Family Planning Commission of Chongqing (2015XMSB63), the Chinese Society of Academic Degrees and Graduate Education (B1-YX20180302-09), the Research and Innovation Project of Graduate Students in Chongqing in 2017 (no. CYB17122 for K.-X.Y.) and the Key Project of Medical Research of Chongqing Municipal Health Bureau (no. 2016ZDXMoo7 for Z.-L.D.).

Manuscript received: $12-07-2018$ Revised manuscript received: 02-18-2019

Accepted for publication: 02-27-2019

Free full manuscript: www.painphysicianjournal.com
Background: Percutaneous endoscopic cervical discectomy has evolved as an efficient, minimally invasive spine surgery for radiculopathy caused by soft and/or osseous foraminal stenosis. Although interlaminar access can be used to resect lateral herniated lesions or osteophytes located in the foramina, with limited operative space, nerve retraction may be unavoidable. This procedure may injure the nerve root and cause postoperative arm pain, numbness, and muscle weakness, especially when the herniation is located in the ventral nerve root or when there is a massive osteophyte in the foramina. However, posterior partial cervical pediculectomy under endoscopy provides a new approach to effectively reduce or even avoid nerve retraction and reduce the potential risk of nerve injury.

Objectives: This report presents a partial pediculectomy approach and compares the clinical outcomes of different surgical methods, including posterior percutaneous endoscopic cervical discectomy (P-PECD) and P-PECD combined with partial pediculectomy

Study Design: This study used a retrospective comparative study design.

Setting: This study took place at the Second Affiliated Hospital of Chongqing Medical University.

Methods: From February 2015 to March 2017, 84 patients with single-level and unilateral soft and/or osseous cervical foraminal stenosis were recruited. Patients were treated with P-PECD (40 patients) and P-PECD combined with partial pediculectomy (44 patients). Postoperative clinical outcomes were assessed using the modified MacNab grading criteria and the Visual Analog Scale (VAS) at different times after surgery. The surgery duration, dosage of postoperative analgesic medication, duration of hospital stay, and postoperative complications were recorded.

Results: The mean duration of the conventional P-PECD surgery was $74.48 \pm 7.08$ minutes, which was significantly longer $(P=0.002)$ than that observed for the P-PECD with partial pediculectomy (66.00 \pm 9.62 minutes). The analgesic dosage in the conventional P-PECD group was significantly higher than that in the partial pediculectomy group $(9.14 \pm 3.07$ units vs. $5.71 \pm 3.41$ units; $P=$ $0.001)$. The hospital stay in the conventional P-PECD group was significantly longer than that in the partial pediculectomy group (3.86 \pm 0.85 days vs. $3.24 \pm 0.83$ days; $P=0.022)$. The VAS scores at 1 day, 3 days, and 7 days after surgery in the conventional P-PECD group were significantly higher than those in the partial pediculectomy group (all $P<0.001$ ). The modified MacNab grading criteria showed no significant difference at each follow-up $(P=1)$. The incidence of complications in the P-PECD with partial pediculectomy group $(2 / 44,4.55 \%)$ was significantly lower than that in the conventional P-PECD group (4/40, 10.0\%), including complications of increased pain, increased numbness, and worsening of muscle weakness.

Limitations: This study is limited by being a retrospective study, and by having a small sample size and a short follow-up period.

Conclusions: As an alternative to the P-PECD surgical technique, P-PECD with partial pediculectomy effectively reduced the postoperative complications and may be preferable when considering the surgery duration, postoperative hospital stay, analgesic dosage, and postoperative VAS score.

Key words: Cervical disc herniation, foraminal stenosis, percutaneous endoscopic cervical discectomy, PECD, P-PECD, partial pediculectomy

Pain Physician 2019: 22:E40-E416 
onventional anterior cervical discectomy and fusion (ACDF) is well recognized as the gold standard surgical treatment for cervical disc herniation, as it was demonstrated to have sufficient decompression and good fusion rates $(1,2)$. However, with the prevalence of ACDF, some procedure-related complications have also been reported in many studies, including the degeneration of adjacent segments, graft subsidence, loss of intervertebral space height, pseudarthrosis, and access-related complications (3-5). The explorations in surgical techniques for avoiding the potential catastrophic complications of ACDF have drawn attention worldwide.

Since the innovation of percutaneous lumbar discectomy by Kambin (7) and Hijikata et al (6), this technique of percutaneous discectomy has been applied to the treatment of disc diseases in the cervical and thoracic spine. For the cervical spine, percutaneous endoscopic cervical discectomy (PECD) was rapidly developed, and one of the conventional PECD procedures is posterior interlaminar access, which has demonstrated efficacy in several studies (8-10).

At present, the posterior percutaneous endoscopic cervical discectomy (P-PECD) procedure is still evolving rapidly. Although interlaminar access may be able to resect lateral herniated lesions or osteophytes located in the foramina, there are still some inevitable complications. For example, considering the limitation of operative space and a more restricted working channel, excessive nerve root retraction could cause short-term postoperative pain, numbness, and muscle weakness when the compression of the ventral or axillary nerve is removed $(9,11-13)$. To overcome the disadvantage caused by intraoperative retraction of the nerve, the PPECD with partial pediculectomy technique was applied and reported by our team, and a total of 84 patients were reviewed in this study to evaluate the technique.

\section{Methods}

\section{Patient Population}

A total of 84 patients with single-level and unilateral soft and/or osseous cervical foraminal stenosis were treated with PECD from February 2015 to March 2017. Forty patients underwent conventional P-PECD (group A), and 44 patients underwent P-PECD with partial pediculectomy (group B). The demographic characteristics of the patients are shown in Table 1. All procedures were performed by the same surgeon.

The inclusion criteria of this study were as follows: (1) foraminal or lateral cervical disc herniation in which the main part of the disc is located lateral to the lateral edge of the spinal cord on magnetic resonance imaging (MRI) and computed tomography (CT) myelography scan; (2) unilateral cervical radiculopathy with arm pain and/or loss of sensory or motor function; (3) craniocaudal disc migration as long as the lateral localization was maintained; and (4) nonresponsive conservative therapy for at least 6 weeks $(8,14)$. The exclusion criteria were (1) a suspected infection or tumor in the cervical spine; (2) multiple cervical disc herniation with severe degeneration; (3) definitive segmental instability; (4) cervical deformities; (5) severe cervical canal stenosis; (6) medial localization of the disc herniation; and (7) neurologic or vascular pathologies mimicking a herniated disc.

\section{Operative Technique}

\section{Conventional P-PECD Group}

All the procedures were mainly in accordance with previous descriptions of the conventional P-PECD techniques $(8,9,15)$. Under general anesthesia, the patient was placed in the prone position on a radiolucent table; the neck was fixed and oriented in slight flexion.

Once the target level of the vertebra was demarcated by fluoroscopy, an 8-mm transverse skin incision was made above the facet joint of the pathological side under x-ray control (approximately $2 \mathrm{~cm}$ lateral from midline) (Fig. 1). A blunt puncture needle was inserted through the incision onto the lamina facet of the target level. A dilator was inserted via the needle, and the endoscopic bevel-ended sheath was inserted via the dilator. The dilator and the puncture needle were removed, and then the endoscope was inserted. Further procedures were performed under endoscopy. Bipolar

Table 1. Patient demographic characteristics.

\begin{tabular}{|c|c|c|c|c|c|c|c|c|}
\hline \multirow{2}{*}{ Group } & \multirow{2}{*}{\begin{tabular}{|l|} 
Age $(\text { yrs })^{*}$ \\
$($ mean \pm SD)
\end{tabular}} & \multirow{2}{*}{\begin{tabular}{|l|} 
Gender \\
(Female/Male)
\end{tabular}} & \multicolumn{3}{|c|}{ Treatment Level } & \multirow{2}{*}{\begin{tabular}{|l|} 
Duration (mos) \\
(mean \pm SD) \\
\end{tabular}} & \multirow{2}{*}{$\begin{array}{l}\text { Hand } \\
\text { Numbness }\end{array}$} & \multirow{2}{*}{$\begin{array}{l}\text { Muscle } \\
\text { Weakness }\end{array}$} \\
\hline & & & C4/5 & C5/6 & C6/7 & & & \\
\hline A & $52.9 \pm 14.3$ & $22 / 18$ & 6 & 20 & 14 & $11.2 \pm 5.6$ & $31 / 40$ & $3 / 40$ \\
\hline B & $55.4 \pm 12.3$ & $24 / 20$ & 4 & 22 & 18 & $10.8 \pm 5.8$ & $34 / 44$ & $2 / 44$ \\
\hline
\end{tabular}

${ }^{*} \mathrm{P}=0.537$. Abbreviations: $\mathrm{SD}$, standard deviation. 
radiofrequency coagulation and grasper were used to clean the soft tissues overlying the facet joint and laminae and to expose the "V-point" structure. Then, burr was used to resect the lower edge of the laminae, expose the ligamentum flavum, and move laterally toward the medial half of the facet. After resecting the target bony structures and the ligamentum flava, the dural sac and nerve root were identified. Mobilization of the nerves and resection of the posteriorly protruded nucleus pulposus were performed. Then, a nerve hook was used to confirm the lateral extent of the foraminotomy, with additional bone resection using a burr or Kerrison rongeur. The exiting nerve root was visualized and traced laterally (Fig 2A-C). Instability must be avoided during the PECD, therefore, the facet joint must be preserved at least $50 \%$, or it may cause instability (16).
The decompression was identified adequately by free mobilization of the nerve root from the proximal part to the distal exiting zone. After all instruments were removed, the incision was sutured closed. All patients were advised to wear a neck collar for at least 3 weeks.

\section{P-PECD with Partial Pediculectomy Group}

Compared with conventional P-PECD, the procedural differences started from establishing the working channel. A 2.0-mm Kirschner wire was inserted through the incision into the lateral mass, which was confirmed to be medial to the margin of the projection of the pedicle via x-ray fluoroscopy. The Kirschner wire was vertically anchored to the lateral mass, making sure to avoid entering the spinal canal. After a sequence of dilation along the Kirschner wire, a trephine





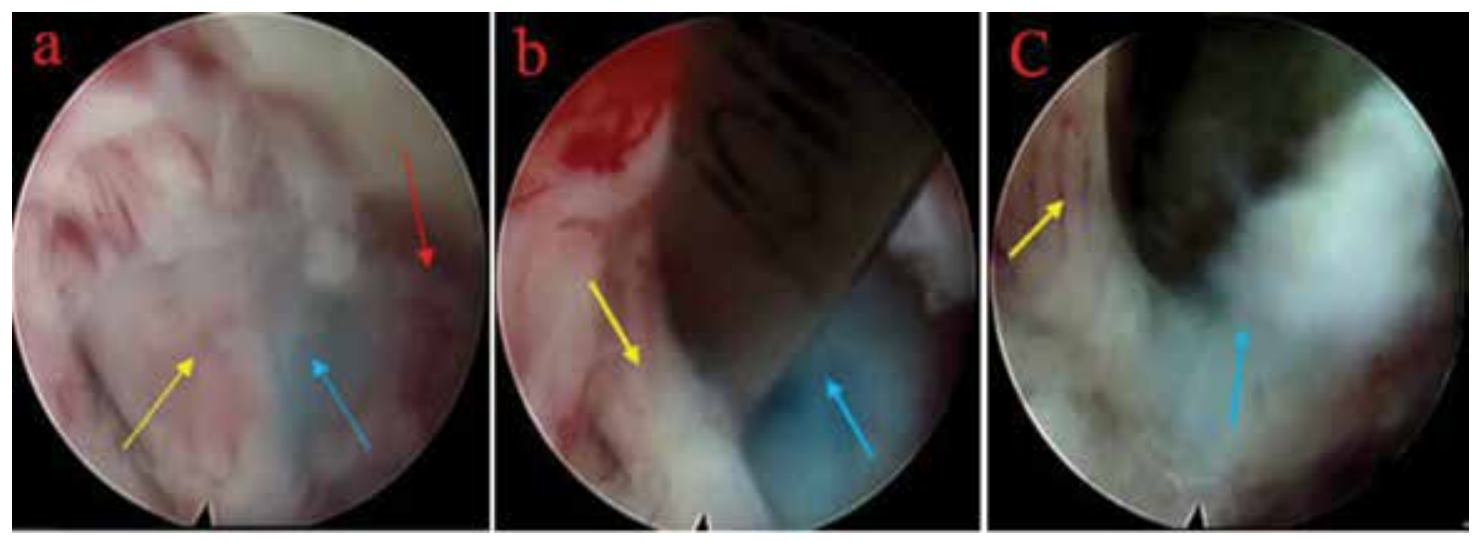

Fig. 2. Endoscopic images of conventional P-PECD. (A) The space between the nerve root (yellow arrow) and the pedicle (red arrow) was very narrow, and the herniated disc (blue arrow) severely squeezes the nerve root. (B) Using the nerve dissector narrowing to probe the herniated disc (blue arrow), the nerve root was excessively retracted (yellow arrow). (C) In the narrow operating space, the nerve (yellow arrow) needs to be significantly retracted to resect the herniated disc (blue arrow).

was used to curve the surface of the lateral mass and double mark the target point (Fig $1 \mathrm{~F}-\mathrm{I}$ ). After the trephine and dilator were removed, the endoscope was inserted into the endoscopic sheath followed by the Kirschner wire $(17,18)$. Then, adequate exposure of the "V-point" and the mark curved by the Kirschner wire was achieved (Fig. 1 A-D). The burr was used to resect the lower edge of the lamina, and exposure to the ligamentum flavum was achieved by moving laterally toward the mark point. Burring would be continued until getting into the spinal canal. Meanwhile, bipolar radiofrequency was used to pretreat the surrounding soft tissue and small blood vessels to more clearly identify the anatomic position of the dural sac, nerve, disc or osteophytes, and pedicle. The location of the pedicle and nerve root axilla were confirmed, and the nerve probe was used to explore the protruded nucleus pulposus of the intervertebral foramen, following the proximal part of the pedicle. The medial-superior part of the proximal pedicle was resected, and the space of the nerve root axilla was increased. Meanwhile, a lowenergy bipolar radiofrequency was used to coagulate the venous plexus and clean the soft tissues and surface of the bone. Then, the endoscope was advanced to reach the axilla of the nerve root. The nucleus pulposus was directly resected, and there was no need to mobilize the nerve root. A nerve probe and burr were more easily used to check and drill the osteophyte located in the foramina through the axilla of the nerve root. The remaining steps were the same as those in conventional group.

\section{Follow-Up}

The radiologic parameters obtained at preoperation and postoperation were evaluated, including $C T$ and MRI scans (Fig. 3,4). The surgery duration, dosage of postoperative analgesia administered for postoperative pain during the hospital stay, and the duration of the hospital stay were recorded. The Visual Analog Scale (VAS) score and the modified MacNab grading criteria were used to evaluate the postoperative outcomes. All complications were recorded.

\section{Statistical Analysis}

The 2-sample t test was used to compare the operative time, the amount of postoperative medicine use, the duration of hospital stay, and the VAS score between these 2 groups. The Fisher exact test was used to compare the rate of excellent and good criteria in these 2 groups. A probability level of 0.05 was considered to be the threshold of significance.

\section{RESULTS}

The surgical findings and postoperative treatment course are shown in Table 2 . The mean surgery duration of the conventional P-PECD group was $74.48 \pm 7.08$ minutes compared with $66.00 \pm 9.62$ minutes for the P-PECD with partial pediculectomy group $(P=0.002)$. The amount of ibuprofen used by patients in the conventional P-PECD group during the hospital stay was significantly higher than that in the P-PECD with partial pediculectomy group $(9.14 \pm 3.07$ units vs. $5.71 \pm 3.41$ units; $P=0.001$ ); however, these results may be influ- 

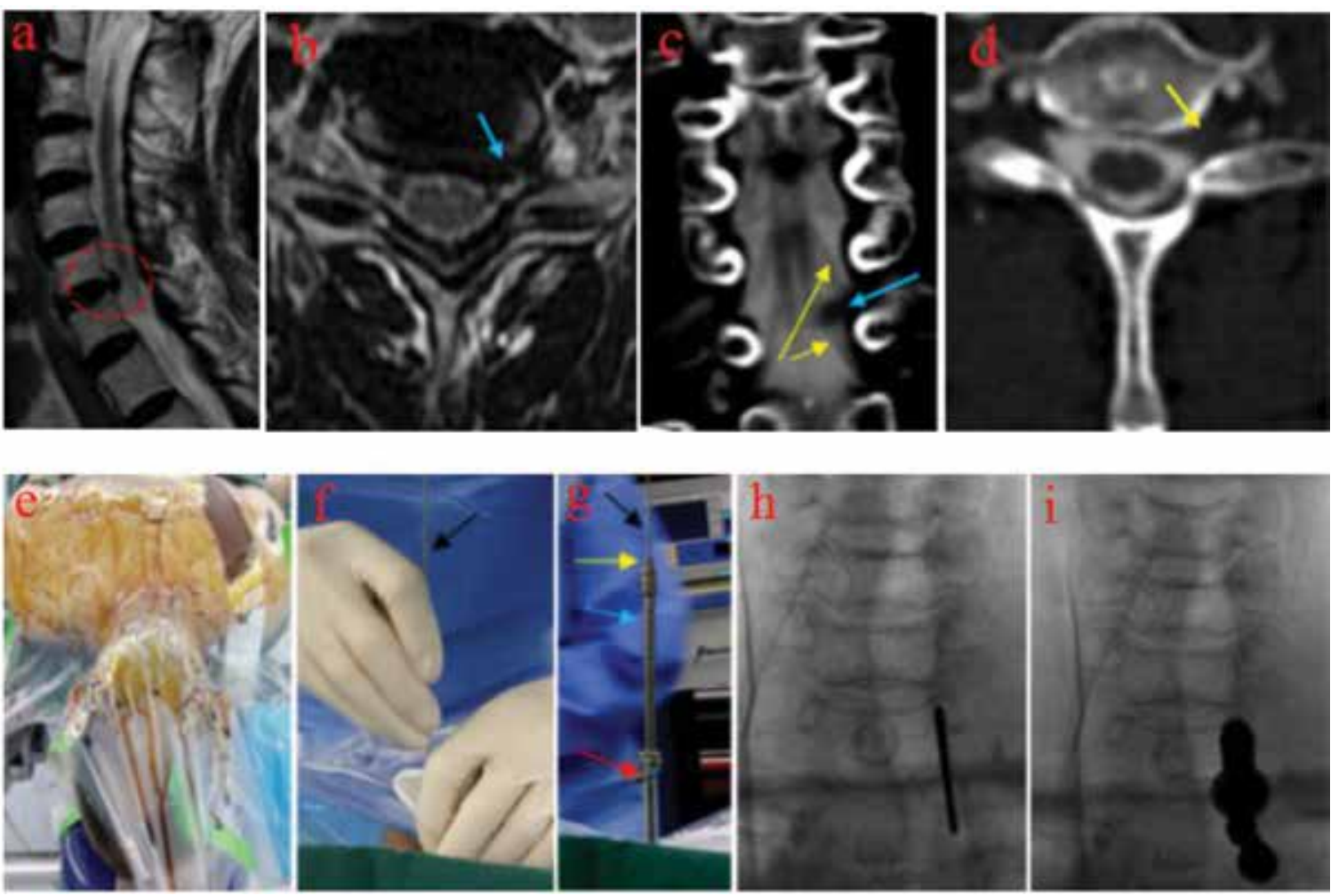

Fig. 3. Preoperative MRI and CTM. a,b. Sagittal and axial MRI scan show the herniated disc compressing the spinal cord (C6/7 level, red circle) and right nerve root of C7 (blue arrow). $c$. A coronal reconstruction of CTM scans shows a herniated disc (blue arrow) compressing nerves and causing deformation (yellow arrow). d. Axial CTM shows a lateral herniated disc compressing the exiting root (yellow arrow). P-PECD with partial pediculectomy technique and anchoring approach. e. Operative position. The patient was placed in a prone position with the neck in slight flexion under combined general anesthesia. $f$. The Kirschner wire(black arrow) was anchoring in the bony surface. g. A sequence of dilation (yellow arrow) along the Kirschner wire (black arrow) was performed and a trephine(blue arrow) was used to curve the surface of the lateral mass and further clean the soft tissues. $h$. X-ray fluoroscopy showed that the Kirschner wire was inserted and located medial-superior part of the projection of the pedicle. $i$. Posterior-anterior fluoroscopy shows the endoscopic sheath was anchored at the medial-superior margin of the projection of the pedicle

enced by the increased length of hospital stay $(3.86 \pm 0.85$ days vs. $3.24 \pm 0.83$ days; $P=0.022$ ).

One patient had no symptom remission after conventional $P$ PECD, which was more likely due to incomplete removal of the nucleus pulposus and incomplete drilling of the foraminal osteophyte. After communicating with the patient, P-PECD with partial pediculectomy was performed and the symptom was resolved.

The preoperative and postoperative VAS score (Fig. 5) and the
Table 2. Surgical findings and postoperative treatment course.

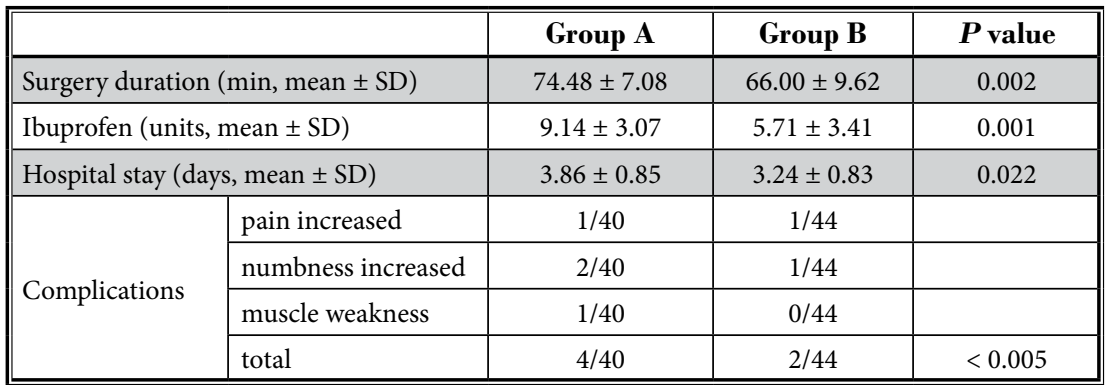

Abbreviations: SD, standard deviation

modified MacNab grading criteria (Fig. 6) were presented. The VAS scores tested at 1 day, 3 days, and 7 days after surgery in the conventional P-PECD group were significantly higher than those in the partial pediculectomy group (all $P<0.001$ ), but these were not significantly different between the 2 

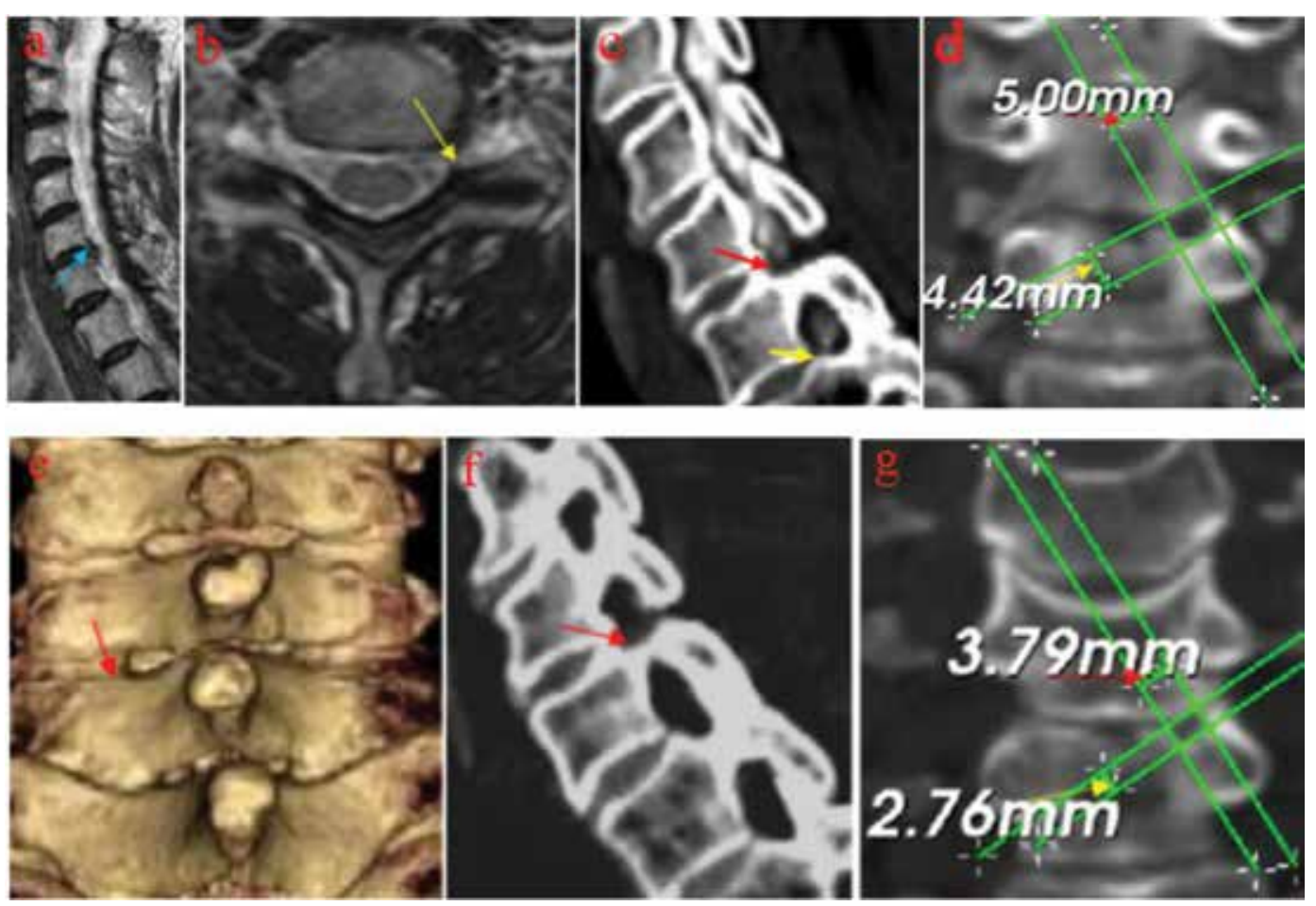

Fig. 4. Postoperative MRI and CT scans at the 6-month follow-up. a,b. The sagittal(blue arrow) and axial MRI(yellow arrow) showed no herniated disc compression of the spinal cord or the nerve root. $c$. The sagittal CT reconstruction shows that C6/7 intervertebral foramen with partial pediculectomy ( red circle) and C7/T1 complete intervertebral foramen(yellow arrow). $d$. The coronal reconstruction of the CTM scan showed that the removed bone range was reduced; the long axis was $5.00 \mathrm{~mm}$ (red arrow) and the short axis was $4.42 \mathrm{~mm}$ (yellow arrow). Follow-up CT and $3 D$ reconstruction images at 3 years postoperation. e. The $3 D$ reconstruction shows that the hole on the lateral mass had basically disappeared (red arrow). $f$. The sagittal CT reconstruction shows that most of the pedicle defects were repaired, and the pedicle had no fracture(red arrow). g. The coronal reconstruction of CT scans shows that the medial-superior partial defect of the pedicle was significantly repaired; the long axis was $3.79 \mathrm{~mm}$ (red arrow) and the short axis was $2.76 \mathrm{~mm}$ (yellow arrow).

groups at the last follow-up $(P>0.05)$. The outcomes of these 2 methods evaluated using the modified MacNab grading criteria showed no significant difference at each follow-up $(P=1)$.

There were no severe complications, such as nerve or spinal cord injury, cervical pedicle fracture, or infection. For the complications, 4 patients in the conventional P-PECD group and 2 patients in the partial pediculectomy group suffered from symptom deterioration. The total rate of complication (6 of 84, $7.14 \%$ ) was acceptable compared with other reports $(9,19-21)$. The incidence of complications in the PPECD with partial pediculectomy group (2/44, 4.55\%) was significantly lower than that in the conventional P-PECD group $(4 / 40,10.0 \%)$. In the conventional PPECD group, pain increased (1 patient), numbness increased (2 patients), and muscle weakness was worse (1 patient). However, in the partial pediculectomy group, only pain increased (1 patient) and numbness increased (1 patient).

\section{Discussion}

ACDF is the standard treatment for cervical disc herniation $(1,2)$. Posterior foraminotomy is considered the most common treatment in lateral pathologies, and the evolution of some cervical minimally invasive techniques are based on this technique, such as microendoscopic foraminotomy and conventional PECD. The purpose of surgical technique improvement should be sufficient decompression and minimizing trauma at the same time. With an incision of $<1 \mathrm{~cm}$, P-PECD may be considered the most minimally invasive surgery compared with conventional microendoscopy and posterior standard foraminotomy. The effectiveness of P-PECD has been well documented in many publications during the last decades $(8,9,15)$. Moreover, compared with the 

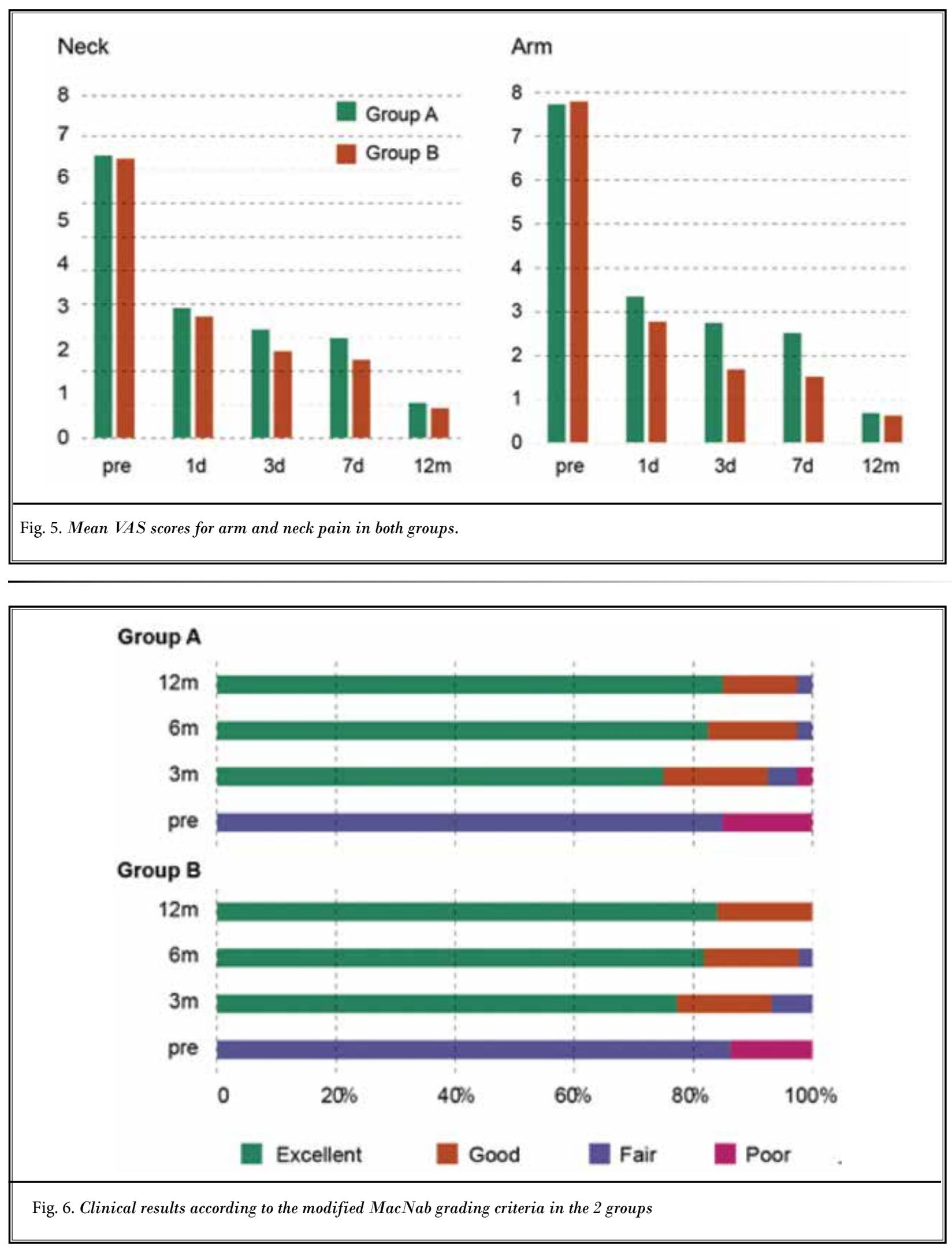
gold standard ACDF technique, these "K-hole" techniques may provide better effectiveness of exposure for decompression of the exiting nerve root caused by lateral disc or osteophytes $(22,23)$.

The procedure of conventional P-PECD is resecting the bone at the medial joint segments and making a hole to reach the lateral edge of the myelon and branching of the spinal nerves. This area is a relatively narrow space for instruments to grab the nucleus pulposus or deal with foraminal stenosis, and the nerve root should be retracted during this procedure. Unlike the standard procedure of P-PECD, P-PECD with the partial pediculectomy provides the following advantages. First, with a landmark curved by a sharp puncture needle, the medial border of the pedicle is achieved before resecting the bone of the lamina and facet joint, which saves time required to expose the target surface of bone. Second, the 2-dimensional vision and anatomic orientation under endoscopic visualization is unfamiliar to surgeons who are accustomed to traditional open surgeries, causing the greatest disadvantage of PECD: a steep learning curve (15). However, the pedicle is an ideal anatomic landmark that can be identified easily under an endoscope. The pedicle is not only the caudal aspect of the foramen but also the lateral border of the cervical spinal canal (24). After drilling, the medialsuperior part of the pedicle can provide sufficient space for surgery to reach the posterior margin of the vertebral body. Moreover, when grabbing the nucleus pulposus or exploring the axilla or shoulder of the nerve root, the tiny space makes it less likely for a surgeon to be able to mobilize the nerve, which could achieve better treatment effectiveness for patients. This is the third advantage.

In this study, we compared conventional P-PECD to P-PECD with partial pediculectomy. The most significant differences between these 2 methods were the surgery duration, dosage of postoperative analgesic treatment for pain, the duration of hospital stay, and the VAS score at postoperative days 1,3 , and 7 . For the surgery duration, the time for surgical positioning and cleaning the soft tissues could be saved using the landmark on the lateral mass. With sufficient space for grabbing the nucleus pulposus or exploring and drilling the osteophytes, there was less bleeding from the epidural venous plexus, which may be another reason for less operative time. For the hospital stay and dosage of analgesic medication, there were significant improvements in P-PECD with partial pediculectomy. This result may be owing to the reduced retraction of the nerve.
The VAS scores at postoperative days 1, 3, and 7 in the P-PECD with partial pediculectomy group were significantly lower than those in the conventional PPECD group, whereas there were no significant differences at later days, and the modified MacNab grading criteria showed no significant difference between the procedures. These data may provide additional support for the improvement of this new technique: faster recovery.

For complications, Choi et al (13) introduced cases of motor palsy after posterior cervical foraminotomy (PCF), and they considered that the $\mathrm{C5}$ nerve root usually covers the entire disc space and therefore needs more excessive retraction than any other nerve roots. Youn et al (11) believe that removal of an extruded disc or bony spur introduces the potential risk of excessive retraction-induced motor palsy and that the risk of transient root injury may be increased due to root retraction without discectomy. Lee et al (25) reported that transient motor weakness and transient sensory changes occurred after PCF, and considered that excessive traction, mechanical injury during drilling, and thermal injury were proposed as causes of weakness or sensory changes after PCF/PECD. In our study, these complications were also inevitable in both groups, but it is worth noting that the symptoms of numbness and aggravation of pain in the partial pediculectomy group were improved approximately 3 days after surgery. In contrast, the conventional group needed approximately 7 days, and muscle strength recovered after 6 months.

The method of resecting the bone of cervical pedicle to reach laterally has been presented using microendoscopy (26), after drilling of the superomedial quadrant of the caudal pedicle provides greater access to the ventral pathology and avoids nerve retraction. Unfortunately, this report was accomplished by microendoscopy surgery. Compared with percutaneous endoscopic surgery, the greatest difference is the clarity of the operative field and visual field. With continuous saline solution irrigation, the generated hydraulic pressure may reduce intraoperative bleeding to provide a clear operative field. In addition, water media could decrease the risk of infection compared with air media. Unlike the static vertical visual field of microendoscopy, PECD with an adjustable visual angle could reduce the possibility of intraoperative iatrogenic injury. In addition, the diameter of the endoscope is $4.3 \mathrm{~mm}$; after resecting the medial-superior of the pedicle, it could provide enough space for the endoscope to be inserted more, making the manipulation safer. 
As a limitation of the sample size and a short follow-up period, the long-term follow-up of this technique needs to be explored in a further study with a larger sample. A precise location of a sharp needle insertion is important for this operation because the drilling range under an endoscope depends on this artificial landmark. The other potential problem is the risk of postoperative cervical pedicle fracture. This outcome was not observed in our study.

\section{Conclusions}

In the era of minimally invasive surgery, sufficient decompression and less trauma are the goals. As a supplement to the described surgical technique of $\mathrm{P}$ PECD, partial pediculectomy is a novel technique for the treatment of radiculopathy. Among the advantages of this technique are reduced retraction of the nerve root and providing a more sufficient space to operate endoscopically compared with the space offered by standard P-PECD. Additionally, shorter surgery duration and hospital stay reduce the use of pain medication. The effectiveness between the standard P-PECD and the P-PECD with partial pediculectomy with a midterm follow-up showed no significant difference.

\section{RefERENCES}

1. Bohlman HH, Emery SE, Goodfellow $\mathrm{DB}$, Jones PK. Robinson anterior cervical discectomy and arthrodesis for cervical radiculopathy. Long-term follow-up of one hundred and twenty-two patients. J Bone Joint Surg Am 1993; 75:1298-1307.

2. Fraser JF, Härtl R. Anterior approaches to fusion of the cervical spine: A metaanalysis of fusion rates. J Neurosurg Spine 2007; 6:298-303.

3. Türeyen K. Disc height loss after anterior cervical microdiscectomy with titanium intervertebral cage fusion. Acta Neurochir (Wien) 2003; 145:565-569.

4. Wang MC, Chan L, Maiman DJ, Kreuter W, Deyo RA. Complications and mortality associated with cervical spine surgery for degenerative disease in the United States. Spine (Phila Pa 1976) 2007; 32:342-347.

5. Jagannathan J, Shaffrey $\mathrm{Cl}$, Oskouian RJ, Dumont AS, Herrold C, Sansur CA, Jane JA. Radiographic and clinical outcomes following single-level anterior cervical discectomy and allograft fusion without plate placement or cervical collar. J Neurosurg Spine 2008; 8:420-428.

6. Hijikata S, Yamagishi M, Nakayama T. Percutaneous nucleotomy: A new treatment method for lumbar disc herniation. J Toden Hosp 1975; 5:5-13.

7. Kambin P. Posterolateral percutaneous suction-excision of herniated lumbar intervertebral discs: Report of interim results. Clin Orthop Relat Res 1986; 207:37-43.

8. Ruetten S, Komp M, Merk H, Godolias G. Full-endoscopic cervical posterior fo- raminotomy for the operation of lateral disc herniations using $5.9-\mathrm{mm}$ endoscopes: A prospective, randomized, controlled study. Spine 2008; 33:940-948.

9. Ruetten S, Komp M, Merk H, Godolias G. A new full-endoscopic technique for cervical posterior foraminotomy in the treatment of lateral disc herniations using 6.9-mm endoscopes: Prospective 2-year results of 87 patients. Minim Invasive Neurosurg 2007; 50:219-226.

10. Kim $\mathrm{CH}$, Shin K-H, Chung CK, Park $\mathrm{SB}, \mathrm{Kim} \mathrm{JH}$. Changes in cervical sagittal alignment after single-level posterior percutaneous endoscopic cervical diskectomy. Global Spine ] 2014; 5:31-38.

11. Youn MS, Shon MH, Seong YJ, Shin JK, Goh TS, Lee JS. Clinical and radiological outcomes of two-level endoscopic posterior cervical foraminotomy. Eur Spine J 2017; 26:2450-2458.

12. Wu PF, Liu BH, Wang B, Li YW, Dai YL, Qing YL, Lv GH. Complications of fullendoscopic versus microendoscopic foraminotomy for cervical radiculopathy: A systematic review and meta-analysis. World Neurosurg 2018; 114:217-227.

13. Choi KC, Ahn Y, Kang BU, Ahn ST, Lee $\mathrm{SH}$. Motor palsy after posterior cervical foraminotomy: Anatomical consideration. World Neurosurg 2013; 79:405.e1405.e4.

14. Olivero WC, Dulebohn SC. Results of halter cervical traction for the treatment of cervical radiculopathy: Retrospective review of 81 patients. Neurosurg Focus 2002; 12: $\mathrm{ECP}$.

15. Ahn Y. Percutaneous endoscopic cervical discectomy using working channel endoscopes. Expert Rev Med Devices 2016; 13:601-610.

16. Zdeblick TA, Zou D, Warden KE, McCabe R, Kunz D, Vanderby R. Cervical stability after foraminotomy. A biomechanical in vitro analysis. J Bone Joint Surg Am 1992; 74:22-27.

17. Yu KX, Chu L, Chen L, Shi L, Deng ZL. A novel posterior trench approach involving percutaneous endoscopic cervical discectomy for central cervical intervertebral disc herniation. Clin Spine Surg 2019; 32:10-17.

18. Liao C, Ren Q, Chu L, Shi L, Yu Q, Yan Z, Yu K, Liu C, Wu W, Xiong Y, Deng Z, Chen L. Modified posterior percutaneous endoscopic cervical discectomy for lateral cervical disc herniation: The vertical anchoring technique. Eur Spine J 2018; 27:1460-1468.

19. Yang J-S, Chu L, Chen L, Chen F, Ke Z-Y, Deng Z-L. Anterior or posterior approach of full-endoscopic cervical discectomy for cervical intervertebral disc herniation? A comparative cohort study. Spine 2014; 39:1743-1750.

20. Winder MJ, Thomas KC. Minimally invasive versus open approach for cervical laminoforaminotomy. Can J Neurol Sci 2011; 38:262-267.

21. Fessler RG, Khoo LT. Minimally invasive cervical microendoscopic foraminotomy: An initial clinical experience. Neurosurgery 2002; 51(5 Suppl):37-45.

22. Raynor RB. Anterior or posterior approach to the cervical spine: An anatomical and radiographic evaluation and 
comparison. Neurosurgery 1983; 12:7-13.

23. Roh SW, Kim DH, Cardoso AC, Fessler RG. Endoscopic foraminotomy using MED system in cadaveric specimens. Spine (Phila Pa 1976) 2000; 25:260-264.

24. Sioutas $\mathrm{G}$, Kapetanakis S. Clinical anatomy and clinical significance of the cer- vical intervertebral foramen: A review. Folia Morphol (Warsz) 2016; 75:143-148.

25. Lee U, Kim CH, Chung CK, Choi Y, Yang $\mathrm{SH}$, Park SB, Hwang SH, Jung JM, Kim KT. The recovery of motor strength after posterior percutaneous endoscopic cervical foraminotomy and discectomy.
World Neurosurg 2018; 115:e532-e538.

26. Gala VC, O’Toole JE, Voyadzis JM, Fessler RG. Posterior minimally invasive approaches for the cervical spine. Orthop Clin North Am 2007; 38:339-349. 